\title{
Denoising of Fingerprint Images using Q-shift Complex Wavelet Transform
}

\author{
V. Sekar \\ Central Instrumentation and \\ Service Laboratory, \\ University of Madras, \\ Maraimali (Guindy) Campus, \\ Chennai- 600025
}

\author{
M. K. Gayathri \\ Central Instrumentation and \\ Service Laboratory, \\ University of Madras, \\ Maraimali (Guindy) Campus, \\ Chennai -600025
}

\author{
D. Nedumaran \\ Central Instrumentation and \\ Service Laboratory, \\ University of Madras, \\ Maraimali (Guindy) Campus, \\ Chennai - 600025
}

\begin{abstract}
In this paper, we present a novel image denoising method using Q-shift with Dual Tree Complex Wavelet Transform (QDTCWT) for denoising fingerprint images. The DTCWT is an over complete wavelet transform with limited redundancy and generates complex coefficients in parallel using a dual tree of wavelet filters. But, low pass delay produces a Hilbert pair relationship between two trees. This is well addressed by Q-shift filters for improving orthogonality and symmetry properties in level 2 and below. QDTCWT have features like linear phase, tight frame, compact spatial support, good frequency domain selectivity with low sidelobe levels, approximate shift invariance, and good directional selectivity in two or more dimensions. This provides the QDTCWT basis mainly useful for de-noising purposes with high degree of shift-invariance and better directionality compared to the other traditional methods. The proposed algorithm has been designed in the MATLAB ${ }^{\mathrm{TM}}$ environment and tested in the fingerprint images obtained from the FVC2004 database for denoising. The performance and efficiency of the algorithm are estimated by calculating various quality metrics and compared with the advanced methods already practiced in fingerprint image denoising. The results of this study revealed that the QDTCWT algorithm is capable of producing high quality finger print images with greater fidelity, high robustness and accuracy over the other traditional denoising methods.
\end{abstract}

\section{General Terms}

Bio-metrics, Fingerprint, Wavelet

\section{Keywords}

Fingerprint, Denoising, Q-shift Dual Tree Complex Wavelet, performance metrics

\section{INTRODUCTION}

Fingerprints are the ridge and furrow pattern on the tip of the finger and are used for personal identification of human subjects [1]. Efficient representation of finger prints information is a critical task, which has been achieved through structural transforms and fast algorithms. Several methods have been used to solve the denoising problems in image analysis and pattern recognition. Generally, the denoising techniques have been categorized into spatial and frequency domain techniques. In spatial domain, adaptive spatial filter is one of the best filtering techniques to restore the heterogeneous pixel characteristics perfectly. Wiener constrained least squares, Lucy Richardson and Blind deconvolution methods are some of the examples of frequency domain filtering techniques. Geman et al [2] introduced a priori constraints deconvolution model using Markov random field (MRF) framework to achieve good estimation and optimization solution in getting acceptable images. Further, the regularization is achieved through denoising process based a priori term [3]. K. Knešaurek and J. Machac [4] implemented Fourier wavelet deconvolution technique in PET images using Butterworth filter and Daubechies wavelet function, which results in $25 \%$ increase in the contrast ratio of lungs with $2 \%$ increase in noise of the restored image. But, fingerprint images containing smooth curves as edges, which cannot be efficiently captured by the normal wavelet transform due to redundant and non-redundant types of wavelets used for denoising. Initially, decimated discrete wavelet transform (DWT) was used in image denoising algorithms, which has non-redundant orthogonal property [5-9]. Due to the non-redundant property, the DWT has the disadvantages of shift in sensitivity, directionality and phase information. Although the stationary wavelet transform (SWT) reduces this problem substantially, but it is computationally expensive [10]. Several other mathematical algorithms [11-13] have also been proposed for solving image processing problems using complex wavelets. The DTCWT is an efficient tool for a variety of image processing applications such as de-noising, edge detection, restoration, enhancement, and compression. In the present work, we have attempted the Q-shift with DTCWT algorithm for denoising fingerprint images.

The remainder of the paper is organized as follows: Section I describes the basics of DTCWT transform, section II deals with Q shift analysis, section III focuses on fingerprint denoising, section IV emphasizes the experimental results, and section V describes the conclusions arrived in this work.

\section{DUAL TREE COMPLEX WAVELET TRANSFORM}

The recent development in wavelet-related research is the design and implementation of 2-D multiscale transforms that represent edges more efficiently than the DWT. Kingsbury's complex dual-tree wavelet transform (DTCWT) is an outstanding example, which generates complex coefficients by using a dual tree of wavelet filters for obtaining their real and imaginary parts separately (Fig.1). The outputs of each tree are down sampled by summing the outputs of the two trees during reconstruction. It is 


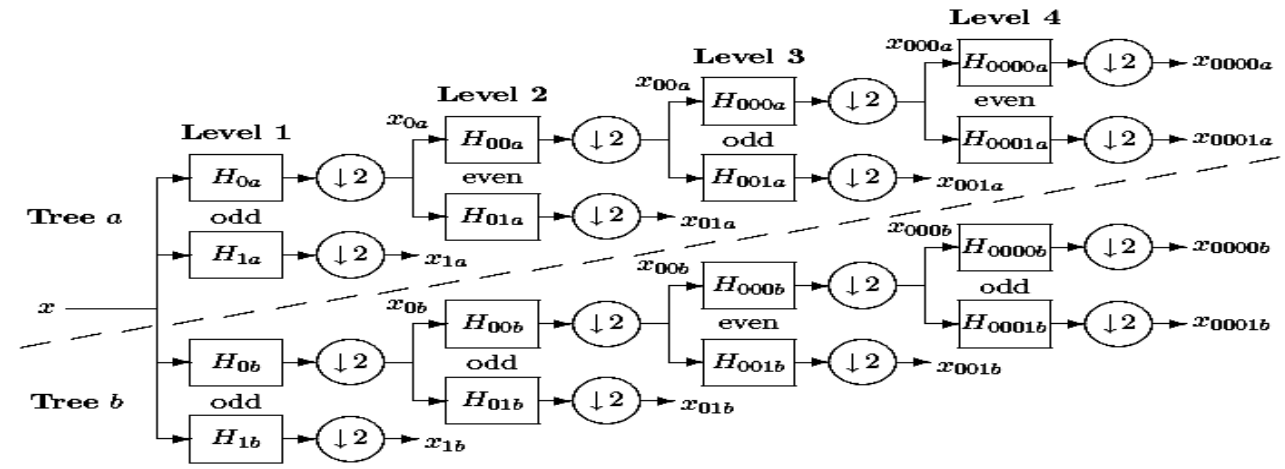

Fig 1 Filter Bank Structure of 4 Level Dual Tree Complex Wavelet

very useful to suppress the aliased components of the signal and achieve approximate shift invariance. Also, it provides the complex wavelet basis mainly useful for de-noising purposes with high degree of shift-invariance and better directionality compared to the real DWT. Further, the DTCWT has solved the problem of inability of perfect reconstruction and good frequency portioning using complex wavelets [14].

For 2-D image signals, filter banks with the pair of trees are applied to the columns and the rows of the image and is represented by

$$
\psi(m, n)=\psi(m) \psi(n)
$$

where $\psi(m)$ is a complex wavelet which gives,

$$
\psi(m)=\psi_{h}(m)+j \psi_{g}(m)
$$

where $\psi_{h}(m)$ and $\psi_{g}(m)$ are both real-valued wavelets. So, $\psi(m, n)$ can be expanded as,

$$
\begin{aligned}
\psi(m, n)= & {\left[\psi_{h}(m)+j \psi_{g}(m)\right]\left[\psi_{h}(n)+j \psi_{g}(n)\right] } \\
= & {\left[\psi_{h}(m) \psi_{h}(n)-\psi_{g}(m) \psi_{g}(n)\right]+} \\
& j\left[\psi_{h}(m) \psi_{g}(n)+\psi_{g}(m) \psi_{h}(n)\right]
\end{aligned}
$$

The sum of two separable wavelets obtained from the real part of the above complex wavelets is given in equation 4 .

$$
\operatorname{Re} a l \text { part }\{\psi(m, n)\}=\psi_{h}(m) \psi_{h}(n)-\psi_{g}(m) \psi_{g}(n)
$$

The complex wavelet $\psi(m)=\psi_{h}(m)+j \psi_{g}(m)$ is approximately analytic therefore $\psi_{h}(m)$ is approximately the Hilbert transform pair of $\psi_{g}(m), \quad$ and hence $\psi_{g}(m)=H\left\{\psi_{h}(t)\right\}$. Also, $\psi_{h}(m) \psi_{h}(n)$ is the HH wavelet of a separable 2-D real wavelet transform implemented using the filters $\left\{H_{o a}, H_{1 a}\right\}$. $\psi_{g}(m) \psi_{g}(n)$ is also the $\mathrm{HH}$ wavelet of a real separable wavelet transform, but it is implemented using the filters $\left\{H_{o b}, H_{1 b}\right\}$.
Repeating the above procedure will result in five more oriented real 2-D wavelets. Then, the six wavelets can be written as:

$$
\begin{gathered}
\psi_{i}(m, n)=\frac{1}{\sqrt{2}}\left(\psi_{1, i}(m, n)-\psi_{2, i}(m, n)\right) \\
\psi_{i+3}(m, n)=\frac{1}{\sqrt{2}}\left(\psi_{1, i}(m, n)-\psi_{2, i}(m, n)\right)
\end{gathered}
$$

where $\mathrm{i}=1,2,3$. For the orthonormal operation the normalization value $1 / \sqrt{2}$ is used. In case of real $2 \mathrm{D}$ filter banks, the three high-pass filters have orientations of $0^{\circ}, 45^{\circ}$ and $90^{\circ}$; for the complex filters, the six subband filters are oriented at $\pm 15^{0}, \pm 45^{0}, \pm 75^{0}$. Fig. 1 shows that there is no link between the 2 trees, so that the parallel implementation of filters is possible during algorithm execution. Also, the first stage of filters is different from other stages. Further, complex arithmetic is not needed in any of these stages. In addition to this, the DTCWT has the following properties, which improves the efficiency of denoising in images.

(i) Approximation of shift invariance.

(ii) Good selectivity and directionality in 2-dimensions.

(iii) Perfect reconstruction (PR) using short linear phase filters.

(iv) Four times redundant for images (4:1).

(v) Efficient order - $\mathrm{N}$ computation - only 4 times the simple DWT.

\section{Q- SHIFT DUAL TREE COMPLEX WAVELET TRANSFORM}

DTCWT approach doesn't address the odd/even filter characteristics due to sub-sampling asymmetry structure and biorthogonal nature of filters sets for wanting of linear phase requirements, which results in non-preservation of energy between the signal and transform domains [14]. These drawbacks have been overcome with the more recent form of dual tree approach known as a Q-shift dual tree [15]. In the Qshift dual tree approach, two sets of filters have been used such as the filters at level 1 , and the filters at all higher levels. The filters beyond level 1 have even length but are no longer strictly linear phase. Extension of Q-shift dual tree in 2D produces three sub images in each of spectral quadrants 1 and 2 giving six 
band-pass sub images of complex coefficients at each level, which are strongly oriented at angles of $\pm 15^{\circ}, \pm 45^{\circ}, \pm 75^{\circ}$. The strong orientation occurs because complex filter can separate positive from negative frequencies vertically and horizontally. The resulting image from transformation shows good rotational invariance, because each image has coefficients from all six directional subbands at the given wavelet level. As a result, the Q-shift dual tree transform retains good shift invariance and directionality properties of the original while improving the sampling structure.

\subsection{Fingerprint Image Denoising}

A fingerprint image consists of non-ridge area, high quality ridge area, and low quality ridge area. It is well known that low quality ridge area in the fingerprint images would cause serious effects, which deteriorate the quality of the image. In order to decrease the effects caused by low quality area, the improved QDTCWT has been applied in the local areas of the fingerprint image. The resulting sub-image is extracted from the original fingerprint image in the complex wavelet transform domain using QDTCWT. Then, according to the characteristics of the subimage data, the denoised fingerprint subimage adaptively spread spectrum and add into the host subimage QDTCWT coefficients.

\subsection{DTCWT Denoising Algorithm}

1. Input the noisy fingerprint image (size 512 x512).

2. Select the window (size is set to $5 \times 5$ )

3. Calculate the forward QDTCWT transform of the selected window data. In our particular example, we calculate four levels of the transform applying reflection boundary conditions.

4. Estimate the noise variance from a median-absolutedeviation estimator applied to the coefficients at the finest scale level. This approach gave a value of 0.084 for the noise standard deviation of the wavelet coefficients.

5. Select the threshold value T using Bays thresholding scheme and apply the soft threshold method level-bylevel to obtain a new array of QDTCWT coefficients.

6. Obtain the denoised image by inverse computation of QDTCWT.

\section{EXPERIMENTAL RESULTS}

The experimentation has been performed on three FVC2004 fingerprint database (DB1_B, DB2_B, DB3_B, ) [15]. We randomly selected 200 fingerprint images from the above database. The study was made in whorl, arch, tent arch, right loop, left loop, and mixed types of fingerprints. To extract the rich details of the fingerprint image, a 4-level QDTCWT is applied to the normalized image. To avoid problems with boundary conditions, the fingerprint image is padded with zero before applying the algorithm. This padding was removed prior to inverse transform. To compare the efficiency of the
QDTCWT algorithm, the fingerprint images were subjected to several other established denoising algorithms such as stationary wavelet transform (SWT) [8], NeighShrink (NS) [16], and ProbShrink (PS) [17]. The original and denoised fingerprint output images obtained from these algorithms are shown in fig. 2 .

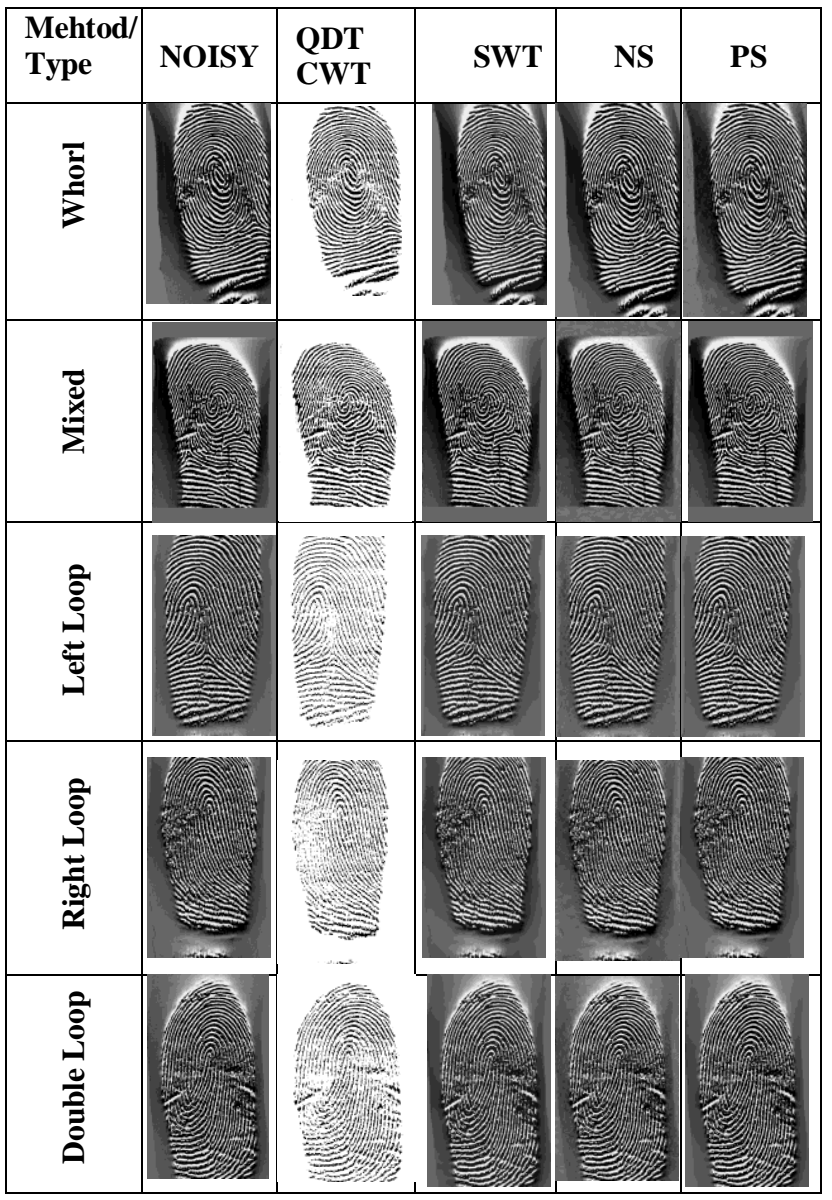

Fig 2 Noisy and Denoised images of five types of fingerprint images using four-different denoising filters

Also, to estimate the performance of the algorithm quantitatively, several traditional performance metrics such as Average Difference (AD), Mean Square Error (MSE), Root Mean Square Error (RMSE), Peak Signal to Noise Ratio (PSNR), Maximum Difference (MD), Normalized Absolute Error (NAE), Signal to Noise Ratio (SNR), Structural Content (SC), Coefficient of Correlation (CoC), Normalized Cross Correlation (NCC), Image Quality Index (IQI), Average Signal to Noise Ratio (ASNR), Image Variance (IV), Noise Standard Deviation (NSD), Effective number of Looks (ENL) and Mean Structural Similarity Index Measure (MSSIM) [18-24] have been calculated. The calculated performance metrics are given in table 1. 
Further, the QDTCWT algorithm concentrates a large amount of effort in preserving the ridge pattern, which plays a critical role in the minutiae extraction algorithm when the quality of the input fingerprint image is poor. To better illustrate our approach, we applied the proposed QDTCWT algorithm in real fingerprint images and the results obtained were analyzed in both qualitative and quantitative points of view. Also, performance of a QDTCWT algorithm was subjectively measured by visual inspection of enhanced images by experts. Fig. 2 visually demonstrates the effectiveness of the proposed algorithm on fingerprint images. The quantitative performance metric values also clearly showed that the QDTCWT algorithm exhibits excellent performance in denoising over the other traditional techniques. The performance metrics calculated are graphically represented in fig 3 . The system level performance of the proposed algorithm is measured in both encryption and decryption part of network cryptography software and the measured value lies within the theoretical limit.

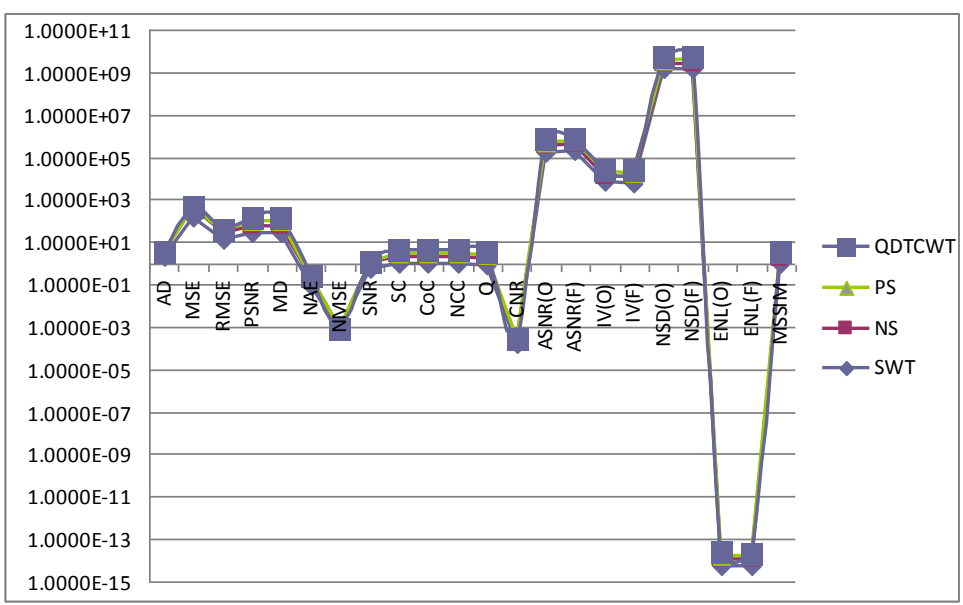

Fig 3 Graphical representation of the performance metrics given in Table 1

Table 1 Performance metrics calculated for the four denoising algorithm for the whorl type fingerprint image

\begin{tabular}{|c|c|c|c|c|}
\hline Parameters & SWT & NS & PS & QDTCWT \\
\hline $\mathrm{AD}$ & 2.000463 & 1.421328 & 0.046488 & 0.046953 \\
\hline MSE & 144.6772 & 294.7043 & 11.36686 & 11.48053 \\
\hline RMSE & 12.02818 & 17.16695 & 3.37147 & 3.38829 \\
\hline PSNR & 27.00491 & 23.46742 & 39.1089 & 39.49999 \\
\hline MD & 27.00491 & 23.46742 & 39.1089 & 39.49999 \\
\hline NAE & 0.087346 & 0.122766 & 0.014653 & 0.014799 \\
\hline SNR & 0.579132 & 0.502006 & 0.035032 & 0.035382 \\
\hline $\mathrm{SC}$ & 1.028819 & 1.029069 & 1.003746 & 1.013783 \\
\hline $\mathrm{CoC}$ & 0.990521 & 0.977373 & 0.999052 & 1.009042 \\
\hline $\mathrm{NCC}$ & 0.981455 & 0.976357 & 0.99776 & 1.007738 \\
\hline IQI & 0.834323 & 0.713396 & 0.898918 & 0.907907 \\
\hline $\operatorname{ASNR}(\mathrm{O})$ & $1.71 \mathrm{E}+05$ & $1.71 \mathrm{E}+05$ & $1.71 \mathrm{E}+05$ & $1.73 \mathrm{E}+05$ \\
\hline $\operatorname{ASNR}(\mathrm{F})$ & $1.87 \mathrm{E}+05$ & $1.84 \mathrm{E}+05$ & $1.72 \mathrm{E}+05$ & $1.74 \mathrm{E}+05$ \\
\hline $\mathrm{IV}(\mathrm{O})$ & $6.49 \mathrm{E}+03$ & $6.49 \mathrm{E}+03$ & $6.49 \mathrm{E}+03$ & $6.59 \mathrm{E}+03$ \\
\hline $\mathrm{IV}(\mathrm{F})$ & $5.69 \mathrm{E}+03$ & $5.79 \mathrm{E}+03$ & $6.45 \mathrm{E}+03$ & $6.55 \mathrm{E}+03$ \\
\hline $\mathrm{NSD}(\mathrm{O})$ & $1.30 \mathrm{E}+09$ & $1.30 \mathrm{E}+09$ & $1.30 \mathrm{E}+09$ & $1.31 \mathrm{E}+09$ \\
\hline NSD(F) & $1.35 \mathrm{E}+09$ & $1.34 \mathrm{E}+09$ & $1.30 \mathrm{E}+09$ & $1.31 \mathrm{E}+09$ \\
\hline ENL(O) & $5.39 \mathrm{E}-15$ & $5.39 \mathrm{E}-15$ & $5.39 \mathrm{E}-15$ & $5.45 \mathrm{E}-15$ \\
\hline$\overline{E N L(F)}$ & $5.17 \mathrm{E}-15$ & $5.23 \mathrm{E}-15$ & $5.38 \mathrm{E}-15$ & $5.43 \mathrm{E}-15$ \\
\hline MSSIM & 0.908134 & 0.846166 & 0.962948 & 0.972577 \\
\hline
\end{tabular}

\section{DISCUSSION AND CONCLUSIONS}

In this work, we have studied fingerprint image denoising using QDTCWT with Bays thresholding technique. The QDTCWT algorithm was developed in the MATLAB 7.1, and tested over 200 different types of fingerprint images. Fifteen performance metrics have been calculated and compared for estimating the efficiency of the QDTCWT algorithm. The results obtained from this study shows that the performance and efficiency of the QDTCWT algorithm exhibits excellent denoising characteristics in preserving the edges (valleys and ridge) patterns very well than the other three methods. Hence, this algorithm might be useful in the areas of minutiae detection, matching, verification and identification. Also, the Q-shift approach increases the accuracy of denoising, which is indicative from the clear ridge patterns (fig.2). Further, small translations do not affect the magnitudes of the complex coefficients due to its shift invariance property, which improve the efficiency of denoising 
in fingerprint images. The soft thresholding method makes the threshold of noisy wavelet coefficients a near-optimal.

Thus, the Q-shift complex wavelet transform provides near-ideal sparsity of representation for both ridges and valleys, which results in more stable magnitude for the ridge and valley patterns. Finally, the QDTCWT with lifting scheme will further increase the computational speed of the algorithm.

\section{ACKNOWLEDGMENTS}

We are most grateful to Prof. Nick Kingsbury, Dept. of Engineering, University of Cambridge, Cambridge, UK for his useful suggestions and for having provided the code for denoising the fingerprint images described in this paper.

\section{REFERENCES}

[1] Maltoni, D., Maio, D., Jain, A.K., and Prabahar,S. 2009. Handbook of Fingerprint Recognition. Springer.

[2] Geman,S., and Geman, D. 1984.Stochastic relaxation, Gibbs distributions and the Bayesian restoration of images. IEEE Trans. Pattern Anal. Mach. Intell.,Vol. 6, No. 6, pp.721-741.

[3] Max Mignotte,. 2006. A Segmentation-Based Regularization Term for Image Deconvolution. IEEE Trans. Image Processing, Vol. 15, No.7.

[4] Knešaurek,K., and Machac,J.2004.Improving detection of small lung nodules in PET imaging using Fourier-wavelet deconvolution.IEEE.

[5] Kingsbury,N.G., and Magarey,J.F.A. 1997. Wavelet Transforms in Image Processing. Proc. First European Conference on Signal Analysis and Prediction, pp.23-24.

[6] Mallat, S.G. 1989. A Theory for Multiresolution Signal Decomposition: The Wavelet Representation. IEEE Trans. PAMI, II, 474-493.

[7] Daubechies,I. 1992. Ten Lectures on Wavelets, CBMSNSF Reg. Con. Series in Appl. Math., SIAM, Philadelphia, FA.

[8] Langis Gagnon,. 1999.Wavelet Filtering of Speckle NoiseSome Numerical Results. Proceedings of the conference Vision Interface, Trois Riviers, Canada.

[9] Pizurica, A., W. Philips,W., Lernahieu,I., Acheroy,M.2000. A Wavelet Based Image Denoising Technique Using Spatial Priors. Proc. ICIP, Vancouver, pp.296-293.

[10] Sekar,V., and Nedumaran,D. 2007. De-noising of fingerprint Images Using Discrete Stationary Wavelet Transform. In Proceedings of the National Symposium on Instrumentation (NSI - 32), India, pp.55-57.

[11] Kingsbury,N., and Magarey,J. 1995.Motion Estimation Using Complex Wavelets. Technical report, Cambridge University Engineering Department, UK.

[12] Kingsbury, N.G. 1998.The Dual-Tree Complex Wavelet Transform: A New Technique for Shift Invariance and Directional Filters. In Proceedings of the IEEE Digital Signal Processing Workshop.
[13] Kingsbury, N.G. 1999. Image Processing with Complex Wavelets. Phil. Trans. Royal Society London.

[14] Kingsbury, N.G. 2001. Complex wavelets for shift invariant analysis and filtering of signals. Journal of Applied and Computational Harmonic Analysis, vol. 10, no 3, pp.234-253.

[15] Kingsbury, N.G. 2003.Design of Q-shift complex wavelets for image processing using frequency domain energy minimization. Proc. IEEE Conf. on Image Processing, Barcelona, Sept 15-17, paper 1199.

[16] NeighShrink (NS), http://bias.csr.unibo.it/fvc2004.

[17] Zhou Dengwen., Cheng Wengang.2008. Image denoising with an optimal threshold and neighbouring window.Pattern Recognition Letters, 29, pp.1694-1697.

[18] Aleksandra Pizurica., and Wilfried Philips. 2006. Estimating the Probability of the presence of a Signal of Interest in Multiresolution Single- and Multiband Image Denoising. IEEE Transactions on image processing, Vol. 15, No. 3.

[19] Christos, P., Loizou, Constantinos, S., Pattichis., Christodoulos I., Christodoulou, Robert, Istepanian,S.H., Marios Pantziaris., and Andrew Nicolaides. 2005.Comparative Evaluation of Despeckle Filtering In Ultrasound Imaging of the Carotid Artery. IEEE Transactions on Ultrasonics, Ferroelectrics, and Frequency control, Vol. 52, No. 10, pp 1653-1669.

[20] Ismail Avcibas., Bulent Sankur., and Khalid Sayood.2002. Statistical evaluation of image quality measures. Journal of Electronic Imaging. Vol. 11(2), pp. 206-223.

[21] Ahmet, M., Eskicioglu., and Paul S. Fisher. 1995. Image Quality Measures and Their Performance. IEEE Transactions on communications. Vol. 43, No. 12, pp. 2959-2965.

[22] Wang, Z., and A. Bovik, A. 2002. A universal image quality index. IEEE Signal Processing Letters. Vol. 9, No. 3, pp. 81-84.

[23] Shnayderman, A., Alexander Gusev., and Ahmet M. Eskicioglu. 2004. A Multidimensional Image Quality Measure Using Singular Value Decomposition. Proceedings of SPIE Image Quality and System Performance. Vol. 5294, pp. 82-92.

[24] Sivakumar, R., and Nedumaran, D. 2010. Speckle Filtering of Ultrasound B-Scan Images - A Comparative Study of Single Scale Spatial Adaptive Filters, Multiscale Filter and Diffusion Filters. International Journal of Engineering and Technology, ISSN: 1793-8236, Vol.2, No.6, pp.514-523. 